This document is the accepted manuscript version of the following article:

Shengelaya, A., Conder, K., \& Müller, K. A. (2019). Signatures of filamentary superconductivity up to $94 \mathrm{~K}$ in tungsten oxide w02.90. Journal of

Superconductivity and Novel Magnetism.

https://doi .org/10.1007/s10948-019-05329-9

\title{
Signatures of Filamentary Superconductivity up to 94 K in Tungsten Oxide $\mathrm{WO}_{2.90}$
}

\author{
A. Shengelaya ${ }^{1,2}$, K. Conder ${ }^{3}$, K. A. Müller ${ }^{4}$ \\ ${ }^{I}$ Department of Physics, Tbilisi State University, Chavchavadze 3, GE-0128 Tbilisi, Georgia \\ ${ }^{2} I v a n e$ Javakhishvili Tbilisi State University, Andronikashvili Institute of Physics, 0177 Tbilisi, Georgia \\ ${ }^{3}$ Laboratory for Multiscale Materials Experiments, Paul Scherrer Institute, 5232 Villigen PSI, Switzerland \\ ${ }^{4}$ IBM Research Laboratory, Säumerstrasse 4, CH-8803 Rüschlikon, Switzerland
}

\begin{abstract}
We report results of the search for possible superconducting state in tungsten oxides $\mathrm{WO}_{3-\mathrm{x}}$ with various oxygen deficiency $0<\mathrm{x}<1$. In samples with one particular composition $\mathrm{WO}_{2.9}$ the signatures of superconductivity with the same transition temperature $T_{\mathrm{c}}=80 \mathrm{~K}$ were registered by means of magnetization measurements. By lithium intercalation the $T_{\mathrm{c}}$ was further increased to $94 \mathrm{~K}$. The observed small superconducting fraction and the absence of clear transition in resistivity measurements indicate that the superconductivity is localized in small regions which do not percolate. Electron Paramagnetic Resonance experiments showed the presence of $\mathrm{W}^{5+}-\mathrm{W}^{5+}$ electron bipolarons in reduced tungsten oxide samples. It is proposed that such bipolarons form and cluster within crystallographic shear planes which exist in the Magnéli phase of $\mathrm{WO}_{2.9}\left(\mathrm{~W}_{20} \mathrm{O}_{58}\right)$ and represent charge-carrier rich quasi-1D stripes or puddles. When decreasing temperature, superconducting state can be established locally in such regions similar to cuprates. The obtained results demonstrate that the Magnéli-type tungsten oxides are promising materials to explore high-temperature superconductivity above liquid nitrogen temperature.
\end{abstract}




\section{Introduction}

The discovery of high-temperature superconductivity (HTS) in cuprates [1] stimulated a huge research activity which resulted in the discovery of many new HTS compounds such as doped fullerenes [2], $\mathrm{MgB}_{2}$ [3] and iron-based superconductors [4]. Despite these exciting developments over a period of three decades, hole-doped cuprates remain the only materials which exhibit superconductivity above the liquid nitrogen boiling temperature at ambient pressure. Here one should note, however, the possible nucleation of superconducting regions with $T_{\mathrm{c}}=90 \mathrm{~K}$ on the surface of Na-doped $\mathrm{WO}_{3}$ crystals reported by Reich and Tsabba [5]. Motivated by this interesting finding, we decided to search for HTS phases in doped tungsten oxides, but instead of Na-doped $\mathrm{WO}_{3}$ we paid attention to oxygen deficient tungsten oxide $\mathrm{WO}_{3-\mathrm{x}}$. Recently we proposed that electron-doped oxygen-deficient tungsten oxide $\mathrm{WO}_{3-\mathrm{x}}$ with so-called the Magnéli phase is a promising material to explore the HTS [6]. It was suggested that such material would be another example of bipolaronic superconductivity similar to cuprates.

The stoichiometric tungsten trioxide $\mathrm{WO}_{3}$ has a perovskite-like crystal structure and is insulating since the $\mathrm{W}^{6+}\left(5 d^{0}\right)$ has an empty d-shell. With oxygen reduction $\mathrm{W}^{5+}\left(5 d^{1}\right)$ ions are induced. In the octahedral oxygen coordination $\mathrm{W}^{5+}$ hosts one $5 d$ electron in a triply degenerate orbit and therefore it is the Jahn-Teller (JT) ion. Upon oxygen reduction, when a sufficient number of charge carriers are doped into $\mathrm{WO}_{3-\mathrm{x}}$, metallic conductivity is established [7]. Oxygen-deficient tungsten oxides $\mathrm{WO}_{3-\mathrm{x}}$ recently attracted considerable attention due to their properties of the efficient light-to-heat conversion, which is important for the photothermal and photocatalysis applications [8]. It was demonstrated that the introduction of oxygen vacancies in stoichiometric $\mathrm{WO}_{3}$ induces local surface plasma resonance, which offers strong photoabsorption in a broad wavelength range [9]. Also it was shown that the Magnéli phase electron-doped $\mathrm{WO}_{2.9}$ has interesting thermoelectric properties [10].

Up to now low temperature properties of tungsten oxides received much less attention. Among few reported studies concerning superconductivity in $\mathrm{WO}_{3-\mathrm{x}}$ there was an observation of filamentary superconductivity below $T_{\mathrm{c}}=3 \mathrm{~K}$ in twin walls of reduced $\mathrm{WO}_{3-\mathrm{x}}$ single crystals with unusually high second critical field $H_{\mathrm{c} 2}$ above 15T [11]. Also a small drop of resistivity which is suppressed by magnetic field was reported in granular thin films of $\mathrm{WO}_{3-\mathrm{x}}$ below $7 \mathrm{~K}$ [12]. Here we report results of the search for a possible superconducting state in $\mathrm{WO}_{3-\mathrm{x}}$ in a wide range of oxygen deficiency $0<\mathrm{x}<1$. In the samples with one 
particular composition $\mathrm{WO}_{2.9}$ the signatures of superconductivity with the same transition temperature $T_{\mathrm{c}}=80 \mathrm{~K}$, but varied superconducting volume fractions were registered by means of magnetization measurements. By lithium intercalation the $T_{\mathrm{c}}$ was further increased to $94 \mathrm{~K}$.

\section{Experimental}

The $\mathrm{WO}_{3-\mathrm{x}}$ samples were prepared by the solid state reaction method starting from $\mathrm{WO}_{3}$ and $\mathrm{WO}_{2}$ reagents in the powder form. The appropriate mixtures of these reagents were ground in an agate mortar, placed in alumina cylindrical crucibles and sealed inside evacuated quartz tubes. The probes were heated to $1000^{\circ} \mathrm{C}$ at a rate of $600^{\circ} \mathrm{C} / \mathrm{h}$ and kept at this temperature for 50 hours with subsequent cooling to the room temperature at $300^{\circ} \mathrm{C} / \mathrm{h}$.

The samples were characterized by powder XRD measurements using a D8 Advance Bruker AXS diffractometers with $\mathrm{Cu} \mathrm{K} \alpha$ radiation. The ac susceptibility and dc magnetization measurements were performed on a Quantum Design physical property measurement system PPMS and magnetic property measurement system MPMS magnetometers, respectively. For the zero-field-cooled dc magnetization measurements the samples were first cooled to low temperature (typically $5 \mathrm{~K}$ ) in a zero magnetic field, then the magnetic field was applied and the magnetization was measured with increasing temperature to $300 \mathrm{~K}$. Thereafter, the temperature was reduced to $5 \mathrm{~K}$ in the same applied field. The fieldcooled magnetization was then measured with increasing temperature. The temperature dependence of the electrical resistivity was measured using the four-probe method. Silver paste was used for the electrical contacts. The EPR measurements were performed at $9.4 \mathrm{GHz}$ using a BRUKER ER-200D spectrometer. The samples were irradiated through slits in the front part of the X-band cavity with a halogen lamp light. Different filters were used to study the wavelength dependence.

\section{Results}

Among the studied $\mathrm{WO}_{3-\mathrm{x}}$ samples with differing oxygen content, in samples with nominal composition $\mathrm{WO}_{2.9}$ we observed anomalies resembling superconductivity in magnetization measurements. The temperature dependence of magnetization measured in a field of 100 Oe with zero-field cooling (ZFC) and field cooling (FC) for $\mathrm{WO}_{2.9}$ (CW33) sample is shown in Fig. 1a. Both ZFC and FC magnetizations strongly decrease below $80 \mathrm{~K}$. 
Such a diamagnetic transition is typical for the superconducting state. The diamagnetic moment below the superconducting transition temperature $T_{\mathrm{c}}=80 \mathrm{~K}$ in the $\mathrm{ZFC}$ and FC measurements originates from the screening supercurrent and the Meissner effects, respectively. Moreover, as one can see from Fig. 1a, below $T_{\mathrm{c}}=80 \mathrm{~K}$, the magnetization is strongly irreversible and $M_{\mathrm{FC}}(\mathrm{T})>M_{\mathrm{ZFC}}(\mathrm{T})$, which is characteristic for type-II superconductors with flux pinning. The shielding volume fraction estimated from ZFC magnetization at $10 \mathrm{~K}$ is $\sim 0.01 \%$, suggesting a filamentary character of superconductivity. It should be emphasized, however, that this estimation is valid only under assumption that the size of superconducting regions $d$ is larger than the London penetration depth $\lambda$. In opposite case, i. e. when $d<<\lambda$, the calculated volume fraction will be modified to larger values. The superconducting transition at $T_{\mathrm{c}}=80 \mathrm{~K}$ was also confirmed by ac susceptibility measurements performed at $H_{\mathrm{ac}}=10 \mathrm{Oe}$ and a frequency of $1000 \mathrm{~Hz}$ as shown in Fig. 1b. Observed superconducting transition was reproduced in a few other samples with the same composition $\mathrm{WO}_{2.9}$ prepared during independent synthesis runs. Some examples are shown in Figs. 1c and 1d. It is important that the superconducting transition temperature was always the same $T_{\mathrm{c}}=80 \mathrm{~K}$ with different shielding volume fractions.
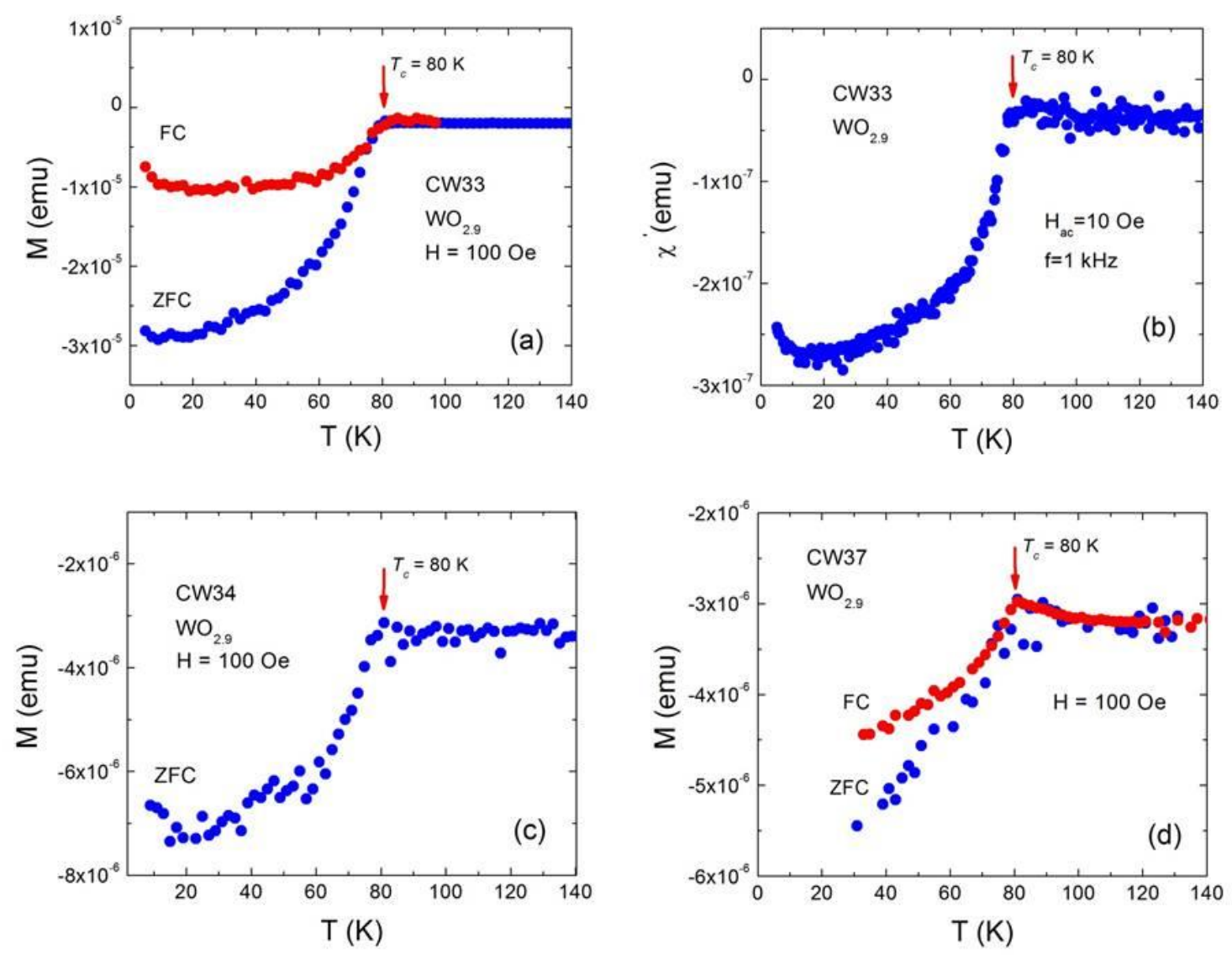
Fig. 1 (a) Temperature dependence of the ZFC and FC dc magnetizations for the $\mathrm{WO}_{2.9}(\mathrm{CW} 33)$ sample in a magnetic field of 100 Oe. (b) Real component of the ac susceptibility as a function of temperature for the $\mathrm{WO}_{2.9}$ (CW33) sample in ac magnetic field of $10 \mathrm{Oe}$ and frequency of $1000 \mathrm{~Hz}$. (c) Temperature dependence of the ZFC dc magnetization for the $\mathrm{WO}_{2.9}$ (CW34) sample in a magnetic field of 100 Oe. (d) Temperature dependence of the ZFC and FC dc magnetizations for the $\mathrm{WO}_{2.9}$ (CW37) sample in a magnetic field of 100 Oe.

Here we would like to note that the drop of the magnetic moment in principle can be also explained by a charge density wave (CDW) transition and was observed for example in the reduced molybdenium oxides [13]. However, in the case of CDW transition there should be no difference between ZFC and FC magnetizations in contrast to our results. As it will be shown below, there are also other results obtained from magnetization measurements which strongly support the superconducting nature of the diamagnetic transitions presented in Fig. 1 .

Interestingly, among several studied $\mathrm{WO}_{3-\mathrm{x}}$ samples with various oxygen contents $(0<\mathrm{x}<1)$, only samples with composition $\mathrm{WO}_{2.9}(\mathrm{x}=0.1)$ showed a superconducting transition. This observation is important as it demonstrates that this transition could not be attributed to some spurious superconducting or magnetic impurity phases present in starting chemicals, since the same starting $\mathrm{WO}_{3}$ and $\mathrm{WO}_{2}$ oxides were used to prepare all $\mathrm{WO}_{3-\mathrm{x}}$ samples. Therefore, it seems that the tungsten oxide with composition $\mathrm{WO}_{2.9}$ is the special one as it hosts a high-temperature superconducting phase. We performed XRD measurements to determine the crystal structure of the obtained $\mathrm{WO}_{2.9}$ samples. It was found that $\mathrm{XRD}$ patterns of these samples correspond to the known monoclinic phase of $\mathrm{WO}_{2.9}$. Actually, the tungsten oxide $\mathrm{WO}_{2.9}$, which can be written with chemical formula $\mathrm{W}_{20} \mathrm{O}_{58}$ is known to be a stoichiometric compound, which belongs to the family of the Magnéli-type oxides with general formula $\mathrm{W}_{\mathrm{n}} \mathrm{O}_{3 \mathrm{n}-2}$ [14]. With oxygen reduction in $\mathrm{WO}_{3}$ the lattice tends to eliminate single oxygen vacancies by the crystal-share process when groups of edge-sharing $\mathrm{WO}_{6}$ octahedra are arranged along some crystallographic planes (shear planes). If these shear planes are parallel and equidistant, a crystalline phase with a defined structure arises as was shown by Magnéli $[15,16]$. Therefore the concept underlying the crystal structure in the Magnéli phases is based on the intrinsic, layered nanostructure defined by crystallographic shear (CS) planes. The $\mathrm{W}_{20} \mathrm{O}_{58}$ phase is one of the most stable and ordered members of the $\mathrm{W}_{\mathrm{n}} \mathrm{O}_{3 \mathrm{n}-2}$ homologous series, where blocks of $2 \mathrm{D}$ corner-sharing $\mathrm{WO}_{6}$ octahedra are mutually connected along CS planes formed by groups of six edge sharing octahedra as illustrated in Fig. 2. 


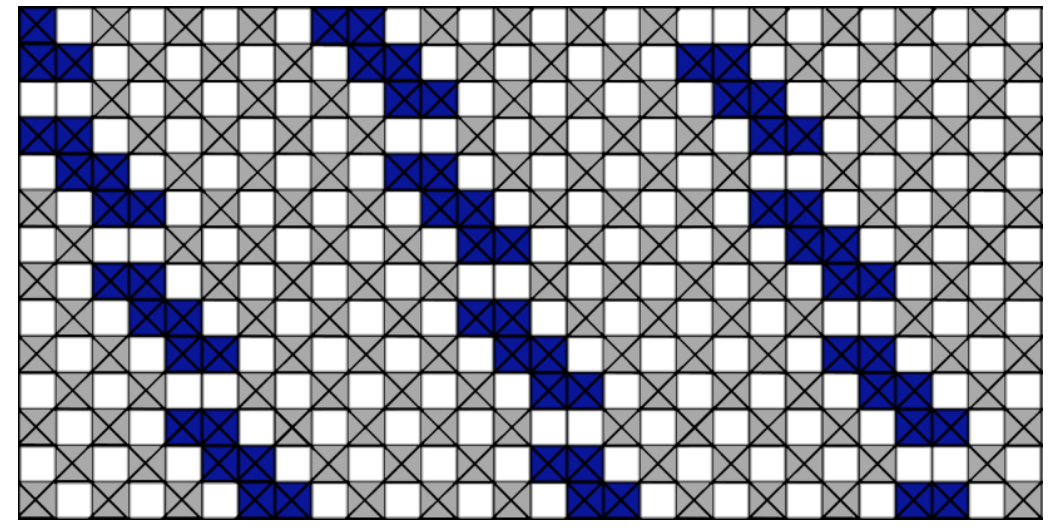

Fig. 2 Idealized crystal structure of the Magnéli phase $\mathrm{WO}_{2.9}\left(\mathrm{~W}_{20} \mathrm{O}_{58}\right)$ projected along [010] with crystallographic shear planes of $\{103\}$ orientation. Blocks of 2D corner-sharing octahedra (grey) from the parent $\mathrm{WO}_{3}$ phase are separated by layers of edge-shared octahedra (blue).

In the $\mathrm{W}_{20} \mathrm{O}_{58}$ phase, $\mathrm{CS}$ planes are parallel to each other with a characteristic period of about $25 \AA$ and $\{103\}$ orientation. It was shown that this oxide has in fact a very narrow homogeneity range close to the theoretical $\mathrm{WO}_{2.9}$ with composition limits of $\mathrm{WO}_{2.89}-\mathrm{WO}_{2.92}$ [17]. This well-defined composition of the $\mathrm{W}_{20} \mathrm{O}_{58}$ phase explains why we observed a single $T_{\mathrm{c}}$ of $80 \mathrm{~K}$ in the studied $\mathrm{WO}_{2.9}$ samples. Also, it is interesting to note that the insulator to metal transition occurs in $\mathrm{WO}_{3-\mathrm{x}}$ at $\mathrm{x}=0.1$ [18]. We think that this is not accidental. Actually, the presence of relatively well-ordered CS planes in the $\mathrm{WO}_{2.9}$ phase as well as sufficient number of charge carriers might be the reason why we observe superconductivity only at this composition.

It was shown that charge carriers in $\mathrm{WO}_{3-\mathrm{x}}$ have polaronic character due to the strong electron-lattice interactions and with decreasing temperature these polarons are paired forming bipolarons $[18,19]$. The most compelling evidence for the existence of bipolarons in oxygen-deficient $\mathrm{WO}_{3-\mathrm{x}}$ was provided by EPR experiments. These experiments showed that no EPR signal from $\mathrm{W}^{5+}$ polarons with spin $\mathrm{S}=1 / 2$ is observed in the reduced tungsten oxide, which indicates that all spins are paired in the ground state. Only after illumination with light of the appropriate energy, bipolarons are broken up into $\mathrm{W}^{5+}$ polarons and an EPR signal can be detected $[19,20]$.

We decided to check the presence of bipolarons in our $\mathrm{WO}_{3-\mathrm{x}}$ samples by performing similar EPR measurements under light illumination. For these experiments single crystals are needed as the EPR signal from $\mathrm{W}^{5+}$ polarons is very anisotropic and will be smeared out in polycrystalline samples. Small $\mathrm{WO}_{3-\mathrm{x}}$ single crystals were grown by heating the $\mathrm{WO}_{3}$ powder in a closed platinum tube at $1370^{\circ} \mathrm{C}$ with subsequent slow cooling $\left(5^{\circ} \mathrm{C} / \mathrm{h}\right)$ to $1270^{\circ} \mathrm{C}$ and 
then fast cooling to the room temperature. Dark single crystals with typical sizes of $0.1-0.5$ $\mathrm{mm}$ were obtained. The dark color of the crystals indicates that they are oxygen-deficient. At the same time they are transparent enough to allow the light penetration during EPR measurements. Fig. 3 shows EPR spectra of $\mathrm{WO}_{3-\mathrm{x}}$ single crystals before and after light illumination. In the crystals cooled in the dark, we did not detect EPR signals from $\mathrm{W}^{5+}$ ions. After light illumination at low temperatures, clear $\mathrm{W}^{5+}$ EPR signals appear. This process was found to be the most effective when the light energy is close to $1 \mathrm{eV}$. We observed several EPR lines since a collection of small single crystals with random orientations was measured. If light is switched off, the EPR signals decay with time due to the polaron recombination into bipolarons with $\mathrm{S}=0$ nonmagnetic ground state.

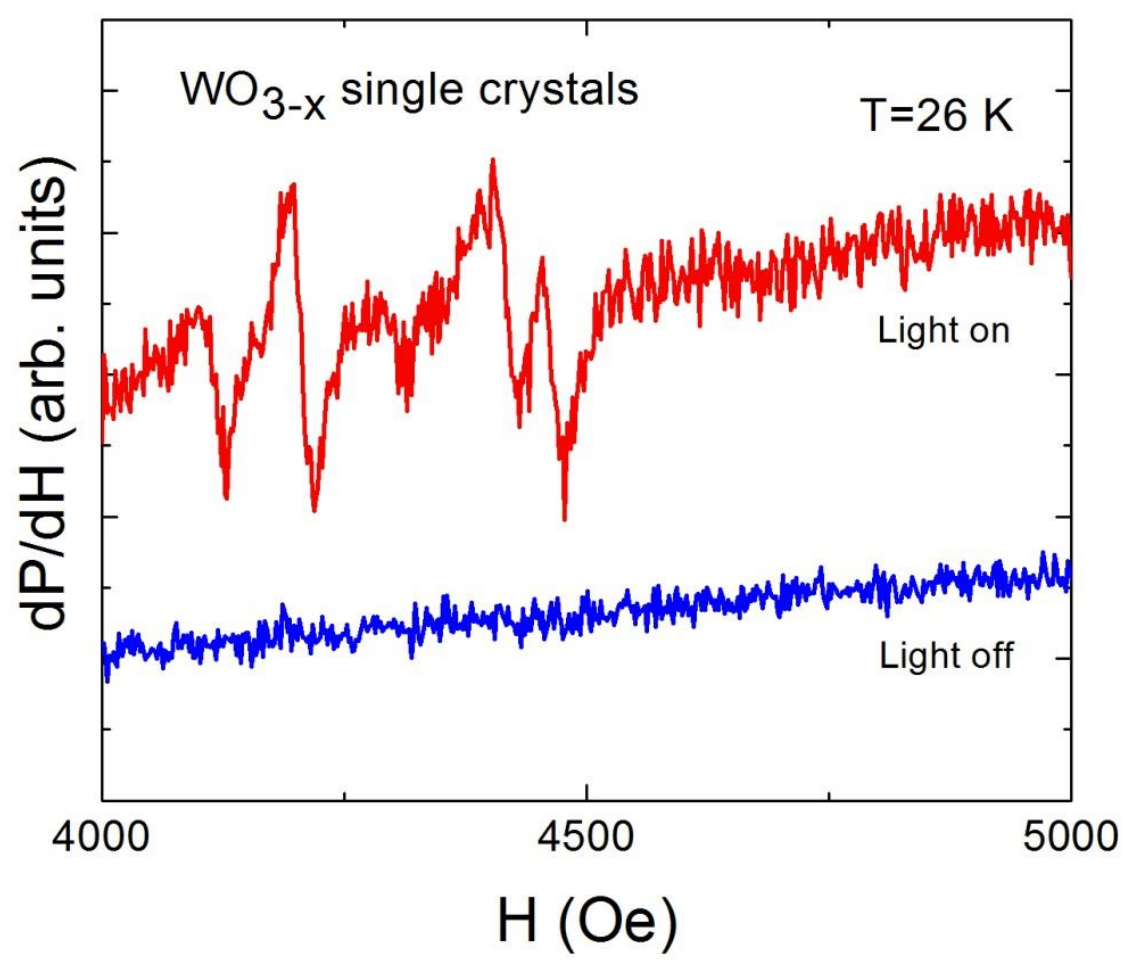

Fig. 3 EPR spectra of $\mathrm{WO}_{3-\mathrm{x}}$ single crystals measured at $T=26 \mathrm{~K}$ before and after light illumination.

These observations are in agreement with the previous reports by Schirmer and Salje $[18,19]$ and confirm the presence of $\mathrm{W}^{5+}-\mathrm{W}^{5+}$ electron bipolarons in oxygen-deficient tungsten oxide. In our opinion such bipolarons are formed in the edge-sharing $\mathrm{WO}_{6}$ octahedra within CS planes of the Magnéli phase $\mathrm{WO}_{3-\mathrm{x}}$ (see Fig. 2). The distance between tungsten ions in the edge-sharing $\mathrm{WO}_{6}$ octahedra is smaller compared to corner shared octahedra of 
the parent $\mathrm{WO}_{3}$ phase. Therefore it is natural to expect that the CS planes with edge-sharing octahedra host the electron bipolarons in $\mathrm{WO}_{3-\mathrm{x}}$. According to this model CS planes form charge-carrier rich metallic stripes or puddles with considerable overlap of $\mathrm{W}^{5+}$ polaron wave-functions [21] and resulting bipolaron formation. With decreasing temperature the superconducting state can be established locally in CS planes, which contain clusters of electron bipolarons. However, the width of the CS planes is very small with thickness of only six edge-sharing $\mathrm{WO}_{6}$ octahedra in the $\mathrm{WO}_{2.9}$ phase as shown in Fig. 2. Therefore in the absence of percolation between the CS planes, the superconductivity will have a filamentary character. This can explain a very small superconducting volume fraction observed in magnetization measurements. We also performed resistivity measurements as a function of temperature to detect the superconducting transition. At low temperatures (below $\sim 250 \mathrm{~K}$ ) resistivity shows metallic behavior in $\mathrm{WO}_{2.9}$ samples, but in contrast to magnetic measurements we did not observe a clear drop of resistivity at $80 \mathrm{~K}$. This indicates the nonpercolative character of superconductivity in these samples.

In order to modify the charge carrier concentration in $\mathrm{WO}_{2.9}$ samples, we performed lithium intercalation by using lithium azide $\mathrm{LiN}_{3}$. The method of thermal decomposition of alkali-metal azides was successfully utilized for the preparation of superconducting alkalimetal doped fullerenes [22]. For lithium intercalation experiments $\mathrm{LiN}_{3}$ and $\mathrm{WO}_{2.9}(\mathrm{CW} 33)$ powders were mixed and loaded in a quartz tube. The tube was connected to a pump and the mixture was slowly heated under the dynamic vacuum. Decomposition of $\mathrm{LiN}_{3}$ started at 230-240 ${ }^{\circ} \mathrm{C}$. After the decomposition was completed, the sample was cooled to room temperature under continuous pumping.

The temperature dependence of the magnetization measured for $\mathrm{Li}$-intercalated $\mathrm{WO}_{2.9}$ (CW33) sample is shown in Fig. 4a. Interestingly, superconducting transition temperature has increased from $80 \mathrm{~K}$ to $94 \mathrm{~K}$ as a result of lithium intercalation. This demonstrates that $\mathrm{WO}_{2.9}$ is indeed an electron doped superconductor. The shielding volume traction remained approximately the same after lithium intercalation. As one can see in Fig. 4a, additional features are seen in both ZFC and FC magnetization curves at $80 \mathrm{~K}$ and $60 \mathrm{~K}$, which indicate the presence of phases with different $T_{\mathrm{c}}$ 's possibly due to inhomogeneous lithium distribution in the sample. Fig. 4b shows magnetization versus magnetic field $M(H)$ dependence measured at $10 \mathrm{~K}$ in lithium doped sample. It demonstrates a behavior typical for type-II superconductor. In low magnetic fields $M(H)$ dependence is linear indicating the Meissner state. The lower critical field $H_{\mathrm{c} 1}$ can be defined as the field in which $M(H)$ starts to deviate from linearity. The value of $H_{\mathrm{c} 1}$ estimated by this method is about $60 \mathrm{Oe}$ at $10 \mathrm{~K}$. 

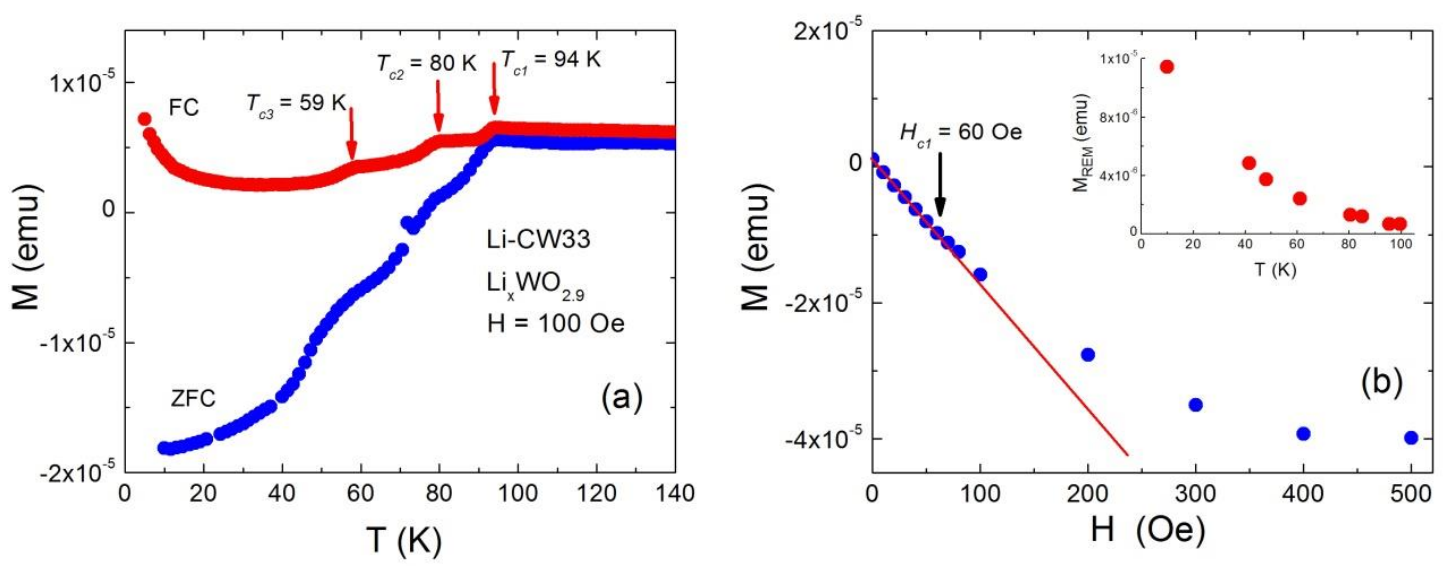

Fig. 4 (a) Temperature dependence of the ZFC and FC dc magnetizations for the lithium intercalated $\mathrm{WO}_{2.9}$ (CW33) sample in a magnetic field of 100 Oe. (b) Magnetization versus applied magnetic field for the lithium intercalated $\mathrm{WO}_{2.9}(\mathrm{CW} 33)$ sample measured at $10 \mathrm{~K}$. The inset displays the remanent magnetization as a function of temperature.

Another hallmark of type-II superconductors is the remanent magnetization $M_{\text {REM }}$ caused by magnetic flux trapping in the vortex state. To measure this quantity the $\mathrm{Li}$ intercalated $\mathrm{WO}_{2.9}$ sample was cooled in a magnetic field from point above $T_{\mathrm{c}}$ to $10 \mathrm{~K}$ and at this temperature the magnetic field was switched off. The remanent magnetization was measured as a function of temperature by warming up the sample. Obtained results are shown

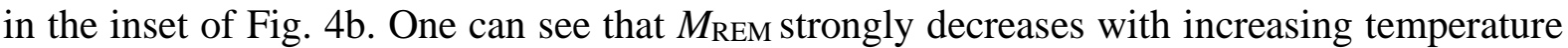
and becomes negligibly small at $95 \mathrm{~K}$, which coincides with the superconducting onset temperature $94 \mathrm{~K}$ determined from the $\mathrm{ZFC} / \mathrm{FC}$ magnetization measurements (see Fig. 4a).

\section{Summary and Conclusions}

To summarize, we observed indications of high-temperature superconductivity at $T_{\mathrm{c}}=$ $80 \mathrm{~K}$ in the Magnéli phase reduced tungsten oxide $\mathrm{WO}_{2.9}\left(\mathrm{~W}_{20} \mathrm{O}_{58}\right)$. The $T_{\mathrm{c}}$ was further increased to $94 \mathrm{~K}$ by lithium intercalation, which shows that the $\mathrm{WO}_{2.9}$ is an electron doped superconductor. By performing EPR measurements under light illumination we confirmed the presence of $\mathrm{W}^{5+}-\mathrm{W}^{5+}$ electron bipolarons in reduced tungsten oxides in agreement with previous reports $[18,19]$. In our opinion such bipolarons form and cluster in the edge-sharing octahedra of CS planes, which represent charge-carrier rich quasi-1D stripes or puddles. Upon cooling, superconducting state can establish locally in CS planes in agreement with our recent proposal [6]. Magnetization measurements showed very small value of the shielding volume fraction, suggesting the filamentary character of superconductivity. Also, in 
resistivity measurements no clear drop of resistivity was found at $T_{\mathrm{c}}=80 \mathrm{~K}$. This can be explained if the size of the superconducting clusters is much smaller than the London penetration length and there is no percolation between them. In such a situation, superconductivity can be detected only in magnetization measurements under the condition that the size of superconducting regions is not too small. Up to now we were able to detect diamagnetic transition in about $20 \%$ of the synthesized $\mathrm{WO}_{2.9}$ samples. The reason for this behavior is probably an intrinsic disorder present in the Magnéli phase [10]. Also there is a possibility that some new phase with a composition of $\mathrm{WO}_{2.9}$ and as yet unknown crystal structure is responsible for the observed superconductivity. In any case, in view of the very small size of the superconducting regions it would be interesting to use scanning imaging techniques such as scanning tunneling microscopy/spectroscopy and scanning SQUID microscopy to identify the regions of $\mathrm{WO}_{2.9}$ samples where superconductivity is localized.

We expect that by improving the coupling and percolation between CS planes, bulk superconductivity and zero resistance state might be achieved due to pair tunneling between electron bipolaron clusters in CS planes. The feasible approach would be to apply hydrostatic or uniaxial pressure to tune the band structure and coupling between CS planes. Also the study of $\mathrm{WO}_{2.9}$ thin films might be promising. Here, we note a recent discovery of superconductivity in the Magnéli-type oxygen-reduced titanium oxide thin films with CS planes where authors concluded that the strain and bipolaronic nature of the charge carriers are responsible for the emerging of superconductivity [23].

In conclusion, the obtained results demonstrate that the Magnéli-type tungsten oxide with a composition $\mathrm{WO}_{2.9}\left(\mathrm{~W}_{20} \mathrm{O}_{58}\right)$ exhibits filamentary, non-percolative superconductivity below $T_{\mathrm{c}}=80 \mathrm{~K}$. Therefore, it is a promising compound for further investigation of HTS above liquid nitrogen temperature, especially for superconductivity applications. One should note that up to now there are no systematic studies of the low temperature magnetic and transport properties of the Magnéli-type oxygen-deficient tungsten oxides. We hope that the results presented in this paper will stimulate research efforts to investigate electronic and magnetic properties in this interesting class of materials. 


\section{Acknowledgements}

We thank H. Keller for his support and interest during this work. We also thank R. Khasanov and F. La Mattina for assistance during the ac susceptibility and EPR measurements. Valuable discussions with A. Bishop, A. Bussmann-Holder, J. Purans and Z. Guguchia are gratefully acknowledged. This work was supported by the Shota Rustavely National Science Foundation of Georgia under grant No. STCU 2017_29.

\section{References:}

1) Bednorz, J. G., Müller, K. A.: Z. Phys. B 64, 189 (1986)

2) Hebard, A. F., Rosseinsky M. J., Haddon R. C., Murphy D. W., Glarum S. H., Palstra T.T.M., Ramirez A. P., Kortan A. R.: Nature 350, 600 (1991)

3) Nagamatsu, J., Nakagawa, N., Muranaka, T., Zenitani, Y., Akimitsu, J.: Nature 410, 63 (2001)

4) Kamihara, Y., Watanabe, T., Hirano, M., Hosono, H.: J. Am. Chem. Soc. 130, 3296 (2008)

5) Reich, S., Tsabba, Y.: Eur. Phys. J. B 9, 1 (1999)

6) Shengelaya, A., Müller, K. A.: J. Supercond. Nov. Magn. 32, 3 (2019)

7) Sahle, W., Nygren, M.: J. Solid State Chem. 48, 15 (1983)

8) Wu, C-M., Naseem, S., Chou, M-H., Wang, J-H., Jian, Y-Q.: Frontiers in Materials 6, 49 (2019)

9) Yan, J., Wang, T., Wu, G., Dai, W., Guan, N., Li, L.: Adv. Mater. 27, 1580 (2015)

10) Kieslich, G., Cerretti, G., Veremchuk, I., Hermann, R.P., Panthöfer, M., Grin, J., Tremel, W.: Phys. Status Solidi A 213, 808 (2016)

11) Aird, A., Salje, E. K. H.: J. Phys. Condens. Matter 10, L377 (1998)

12) Kopelevich, Y., da Silva, R.R., Camargo, B.C.: Physica C 514, 237 (2015) 
13) Schlenker, C., Dumas, J., Escribe-filippini, C., Guyot, H., Marcus, J., Fourcaudot, G.: Phil. Mag. B. 52, 643 (1985)

14) Bursill, L.A., Hyde, B. G.: J. Solid State Chem. 4, 430 (1972)

15) Magnéli, A.: Arkiv. Kemi 1, 223 (1949)

16) Magnéli, A., Blomberg-Hansson, B., Kihlborg, L., Sundkvist, G.: Acta Chem. Scand., 9, $1382(1955)$

17) Marucco, J.-F., Gerdanian, P., Dodé, M.: J. Chim. Phys. 66, 674 (1969)

18) Salje, E. K. H., Eur. J. Solid State Inorg. Chem. 31, 805 (1994)

19) Schirmer, O. F., Salje, E.: J. Phys. C. 13, 1067 (1980)

20) Gazzinelli, R., Schirmer, O. F.: J. Phys. C. 10, L145 (1977)

21) Salje, E., Hoppmann, G.: Phil. Mag. B. 43, 105 (1981)

22) Bensebaa, F., Xiang, B., Kevan, L.: J. Phys. Chem. 96, 6118 (1992)

23) Yoshimatsu, K., Sakata, O., Ohtomo, A.: Sci. Rep. 7, 12544 (2017) 